\title{
Formoterol is more effective than salmeterol in suppressing neutrophil reactivity
}

\author{
To the Editor:
}

The research question addressed in the current study was: do the long-acting $\beta_{2}$-adrenoreceptor agonists (LABAs) formoterol (full agonist) and salmeterol (partial agonist) possess comparable suppressive effects on human neutrophils?

Human blood neutrophils were exposed to the test agents at concentrations of 1, 10 and $100 \mathrm{nM}$ followed by addition of the chemoattractant $N$-formyl-L-methionyl-L-leucyl-L-phenylalanine $(1 \mu \mathrm{M})$ and measurement of generation of reactive oxygen species (ROS), leukotriene $\mathrm{B}_{4}\left(\mathrm{LTB}_{4}\right)$, cAMP and release of elastase using a combination of chemiluminescence, ELISA and colourimetric procedures.

Treatment of neutrophils with formoterol, but not salmeterol, resulted in statistically significant $(\mathrm{p}<0.05)$, dose-related suppression of the production of ROS and $\mathrm{LTB}_{4}(10-100 \mathrm{nM})$ as well as release of elastase (1-100 nM). These inhibitory effects of formoterol on neutrophil reactivity were associated with significant increases $(\mathrm{p}<0.05)$ in intracellular cAMP.

These findings may partly explain the apparently superior efficacy of the formoterol/budesonide combination relative to that of salmeterol/fluticasone in reducing the frequency of exacerbations in patients with chronic obstructive pulmonary disease.

Chronic obstructive pulmonary disease (COPD) is characterised by chronic inflammation of small airways, progressive airflow obstruction and recurrent acute exacerbations [1]. Repeated exacerbations accelerate the decline in forced expiratory volume in $1 \mathrm{~s}\left(\mathrm{FEV}_{1}\right)$, and contribute to the morbidity and mortality associated with this disorder [1]. Airway inflammation in COPD is mediated by diverse mechanisms involving cell types such as $\mathrm{CD}^{+} \mathrm{T}$-lymphocytes, macrophages and particularly neutrophils [1].

Notwithstanding cessation of smoking, combined bronchodilator and anti-inflammatory strategies, most notably with long-acting $\beta_{2}$-agonists (LABAs) and inhaled corticosteroids (ICS), represent the cornerstone of therapy of moderate-to-severe COPD [2]. Guidelines for the management of COPD, such as the Global Initiative for Chronic Obstructive Lung Disease report and UK National Institute for Health and Care Excellence guidelines, recommend LABA monotherapy for less severe disease and combination therapy for more advanced disease. ICS/LABA combination therapies, most commonly fluticasone/salmeterol and budesonide/formoterol, have demonstrated significant efficacy in halting the progression of COPD, and reducing the number of exacerbations per year, as well as the rate of decline in FEV1, in the setting of improved symptoms and quality of life [1-4].

Targeting the pro-inflammatory actions of neutrophils is considered to be a key strategy in the therapy of COPD [1]. However, little is known about either the mechanisms by which this may be achieved with LABA/ICS combination therapy or about the most efficacious combinations of these agents. With this in mind, and given the relative insensitivity of neutrophils to corticosteroids [5], the current study was undertaken with the primary objective of comparing the suppressive effects of two commonly used LABAs, formoterol and salmeterol, on human neutrophils in vitro and to determine the potential role of cAMP in mediating these activities.

Formoterol fumarate dehydrate and salmeterol base were obtained from Sigma-Aldrich (Pty) Ltd (Johannesburg, South Africa) and GlaxoSmithKline (Stevenage, UK) respectively. Both agents were dissolved to a stock concentration of $10 \mathrm{mM}$ in dimethylsulfoxide (DMSO) and used at final concentrations of 1,10 and $100 \mathrm{nM}$ in most of the assays described here. The final concentration of DMSO in each assay was $0.1 \%$ and solvent controls were included in all assays. Unless indicated, all other chemicals and reagents were purchased from Sigma-Aldrich.

The study was approved by the Faculty of Health Sciences Research Ethics Committee of the University of Pretoria (Pretoria, South Africa) and prior informed consent was obtained from all blood donors. 
Neutrophils were isolated from heparinised venous blood ( 5 units of preservative-free heparin per $\mathrm{mL}$ ) from nonsmoking healthy adult volunteers by centrifugation on Histopaque-1077 cushions at $400 \times \mathrm{g}$ for $25 \mathrm{~min}$ at room temperature. The resultant pellets were suspended in PBS (0.15 M, pH 7.4) and sedimented with $3 \%$ gelatine to remove most of the erythrocytes. Following centrifugation $\left(280 \times \mathrm{g}\right.$ at $10^{\circ} \mathrm{C}$ for $\left.10 \mathrm{~min}\right)$, residual erythrocytes in the cell pellets were removed by selective lysis by exposure to $0.83 \%$ ammonium chloride at $4^{\circ} \mathrm{C}$ for $10 \mathrm{~min}$. The neutrophils, which were routinely of high purity $(>90 \%)$ and viability (>95\%), were then resuspended to $1 \times 10^{7}$ cells $\cdot \mathrm{mL}^{-1}$ in PBS, and held on ice until used. In the various assays described below, neutrophils were activated with the chemoattractant $N$-formyl-L-methionylL-leucyl-L-phenylalanine (fMLP) $(1 \mu \mathrm{M}$ final) alone with the exception of the assay of elastase release, for which fMLP was used in combination with cytochalasin B (CB) $(1 \mu \mathrm{M})$.

The generation of ROS was measured using a luminol (5-amino-2,3-dihydro-1,4-phthalazinedione)-enhanced chemiluminescence (LECL) procedure. Briefly, neutrophils $\left(10^{6}\right.$ cells) were pre-incubated for $10 \mathrm{~min}$ at $37^{\circ} \mathrm{C}$ in $900 \mu \mathrm{L}$ Hanks' balanced salt solution (HBSS) (indicator free) (Highveld Biological (Pty) Ltd, Johannesburg) containing luminol at a final concentration of $0.1 \mathrm{mM}$. Following pre-incubation, the test $\beta_{2}$-agonists (1$100 \mathrm{nM}$ ) or solvent control were added to the cells, followed after $20 \mathrm{~s}$ by fMLP and LECL responses recorded using a Lumac Biocounter (model 1020; Lumac Systems Inc., Titusville, FL, USA).

Neutrophil degranulation was measured according to the extent of release of the primary granule enzyme, elastase. Neutrophils were incubated at a concentration of $2 \times 10^{6} \mathrm{~mL}^{-1}$ in $\mathrm{HBSS}$ for $10 \mathrm{~min}$ at $37^{\circ} \mathrm{C}$ followed by sequential addition of the test $\beta_{2}$-agonists $(1-100 \mathrm{nM})$ and, $20 \mathrm{~s}$ later, by fMLP/CB $(1 / 1 \mu \mathrm{M})$. The tubes were incubated for $10 \mathrm{~min}$ at $37^{\circ} \mathrm{C}$ then transferred to an ice bath, followed by centrifugation at $400 \times \mathrm{g}$ for $5 \mathrm{~min}$ to pellet the cells, and the supernatants decanted and assayed for elastase using a micromodification of a standard colourimetric procedure $[6,7]$. The results are expressed as milliunits of enzyme per $10^{7}$ cells.

Leukotriene $\mathrm{B}_{4}\left(\mathrm{LTB}_{4}\right)$ and cAMP were measured using competitive binding immunoassay procedures (Correlate EIA; Assay Designs Inc., Ann Arbor, MI, USA). In the case of $\mathrm{LTB}_{4}$, neutrophils $\left(2 \times 10^{6} \mathrm{~mL}^{-1}\right)$ were pre-incubated for $10 \mathrm{~min}$ at $37^{\circ} \mathrm{C}$ followed by addition of the test agents (10 and $\left.100 \mathrm{nM}\right)$ and fMLP $20 \mathrm{~s}$ apart. Following $5 \mathrm{~min}$ of incubation at $37^{\circ} \mathrm{C}$, the reactions were terminated by the addition of an equal volume of ice-cold HBSS and $\mathrm{LTB}_{4}$ measured in the cell-free supernatants. The results are expressed as picograms of $\mathrm{LTB}_{4}$ per $\mathrm{mL}$.

In the case of cAMP, following pre-incubation of neutrophils $\left(2 \times 10^{6} \mathrm{~mL}^{-1}\right)$ for $10 \mathrm{~min}$ at $37^{\circ} \mathrm{C}$, the cells were treated with the $\beta_{2}$-agonists $(10$ and $100 \mathrm{nM}$ ) for $20 \mathrm{~s}$, after which the reactions were terminated as above. Following centrifugation, the supernatants were discarded and cAMP extracted from the cell pellets by addition of $0.1 \mathrm{M}$ hydrochloric acid for $30 \mathrm{~min}$ followed by centrifugation to remove cell debris. The supernatants assayed for cAMP and the results expressed as picomoles of cAMP per mL.

The results of each series of experiments using neutrophils from four to eight different donors were analysed using the Mann-Whitney U-test for comparison of nonparametric data and are presented as the median values with interquartile ranges.

Results are shown in figure 1a and b for ROS and elastase, respectively. Treatment of neutrophils with formoterol caused statistically significant inhibition of the generation of ROS by fMLP-activated neutrophils, as well as release of elastase by cells activated with fMLP/CB at concentrations of 10-100 and 1-100 nM, respectively $(\mathrm{p}<0.05)$. Salmeterol had no significant effect on either ROS generation or elastase release.

Results are shown in figure $2 \mathrm{a}$ and $\mathrm{b}$ for $\mathrm{LTB}_{4}$ and cAMP respectively. Treatment of neutrophils with formoterol, but not salmeterol, at concentrations of 10 and $100 \mathrm{nM}$ resulted in significant $(\mathrm{p}<0.05)$ dose-related inhibition of production of $\mathrm{LTB}_{4}$ by fMLP-activated neutrophils. Brief treatment $(20 \mathrm{~s})$ of neutrophils with formoterol at both concentrations tested $(10$ and $100 \mathrm{nM})$ resulted in significant $(\mathrm{p}<0.05)$ increases in neutrophil cAMP, which were maximal at $100 \mathrm{nM}$, while salmeterol was ineffective.

The findings of the current study demonstrate that formoterol, a full agonist of $\beta_{2}$-adrenoreceptors ( $\beta_{2} A R s$ ), is significantly more potent than the partial agonist, salmeterol, with respect to suppression of neutrophil reactivity in vitro. At the concentrations tested, the inhibitory effects of formoterol on the generation of ROS and $\mathrm{LTB}_{4}$ by chemoattractant-activated neutrophils, as well as release of elastase from these cells, were maximal at a concentration of $10 \mathrm{nM}$. This is relevant, as following inhalation of a single $24-\mu \mathrm{g}$ dose of formoterol, the local concentration of this $\beta_{2}$-agonist in the airways has been estimated to reach $11 \mathrm{nM}$ [6]. The suppressive effects of formoterol on neutrophil reactivity were associated with significant increases in intracellular cAMP consistent with activation of $\beta_{2}$ ARs, which are expressed on human neutrophils [8]. The mechanism by which formoterol, as well as that of indacaterol, an ultra-LABA with nearly full $\beta_{2}$-agonist activity, suppress neutrophil reactivity appears to be achieved by cAMP-dependent protein kinase 
a)

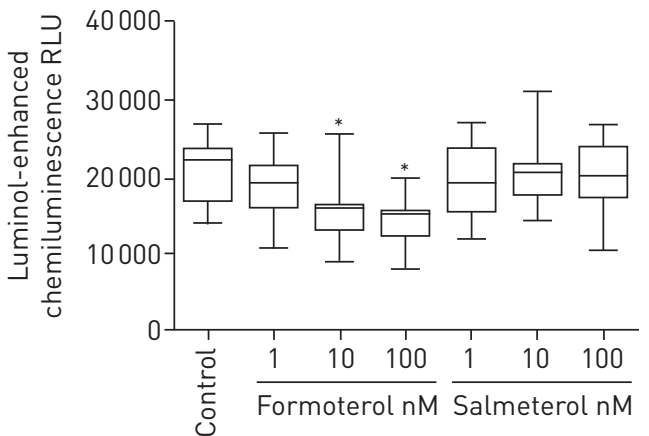

b)

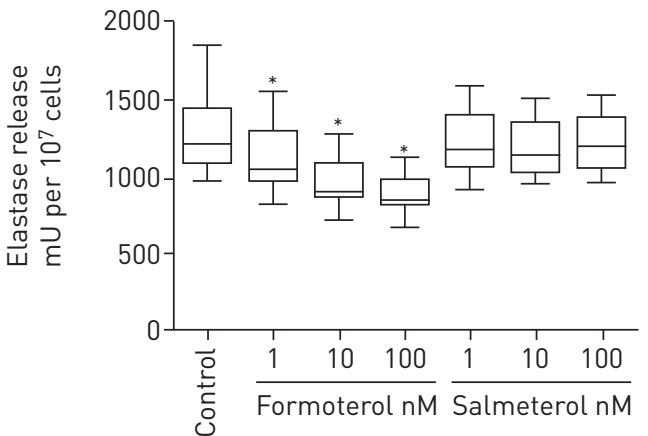

FIGURE 1 The effects of formoterol and salmeterol (1-100 nM) on the a) generation of superoxide and b) release of elastase following activation of neutrophils with $\mathrm{N}$-formyl-L-methionyl-L-leucyl-L-phenylalanine ( $f M L P)(1 \mu \mathrm{M})$ alone, or in combination with cytochalasin B (CB) $(1 \mu \mathrm{M})$ in the case of elastase release $(n=4-5)$. The median values (interquartile range) for the responses of control (drug-free), unstimulated neutrophils for superoxide generation and release of elastase were 1211 (1081-2695) relative light units (RLU) and 155 (81-291) $\mathrm{mU}$ enzyme per $10^{7}$ cells, respectively; the values for the corresponding drug-free control systems activated with fMLP or fMLP/CB were 22056 (17458-23637) RLU and $1214(1088-1409) \mathrm{mU}$ per $10^{7}$ cells. *: $p<0.05$ for comparison of formoterol with the corresponding drug-free control system.

(PKA)-mediated restoration of calcium homeostasis following receptor-mediated activation of the cells with calcium-mobilising stimuli $[6,7]$.

In addition to neutrophils $[6,7,9,10]$, LABAs have also been reported to target other types of $\beta_{2}$ AR-expressing inflammatory and structural cells, including dendritic cells [11] and airway smooth muscle cells [12]. Although the clinical significance of these anti-inflammatory properties of LABAs remains contentious, the beneficial synergistic interactions of these agents with corticosteroids may be of greater relevance. These have been reviewed in detail recently [9] and include LABA-mediated: 1) potentiation of glucocorticoid receptor anti-inflammatory gene transcription; and 2) antagonism of the $\mathrm{LTB}_{4}$-mediated proapoptotic effects of corticosteroids on neutrophils and monocytes. Both activities are achieved by cAMP/PKA-dependent mechanisms. In addition, corticosteroids have also been reported to augment $\beta_{2} \mathrm{AR}$ signalling by increasing the numbers of $\beta_{2} \mathrm{ARs}$ on target cells, as well as by increasing the efficacy of $\beta_{2} A R / G_{s}$-protein subunit coupling (reviewed by THERON et al. [9]).

In apparent contrast to the findings of the current study, STRANDBERG et al. [13] reported that exposure of neutrophils from healthy humans to lipopolysaccharide (LPS) in vitro in the presence of therapeutically relevant concentrations and higher of formoterol for $8 \mathrm{~h}$ resulted in significant increases in the production of the cytokine interleukin (IL)-6 and the chemokines CXCL1 (GRO $\alpha$ ) and CXCL8 (IL-8). Shorter exposure of neutrophils to the LABA (30-60 min) resulted in upregulation of expression of the chemokine receptors CXCR1 and CXCR2, while chemotaxis in response to CXCL8 was unaffected. These observations, taken together with the findings of the current study, suggest that the inflammatory activities
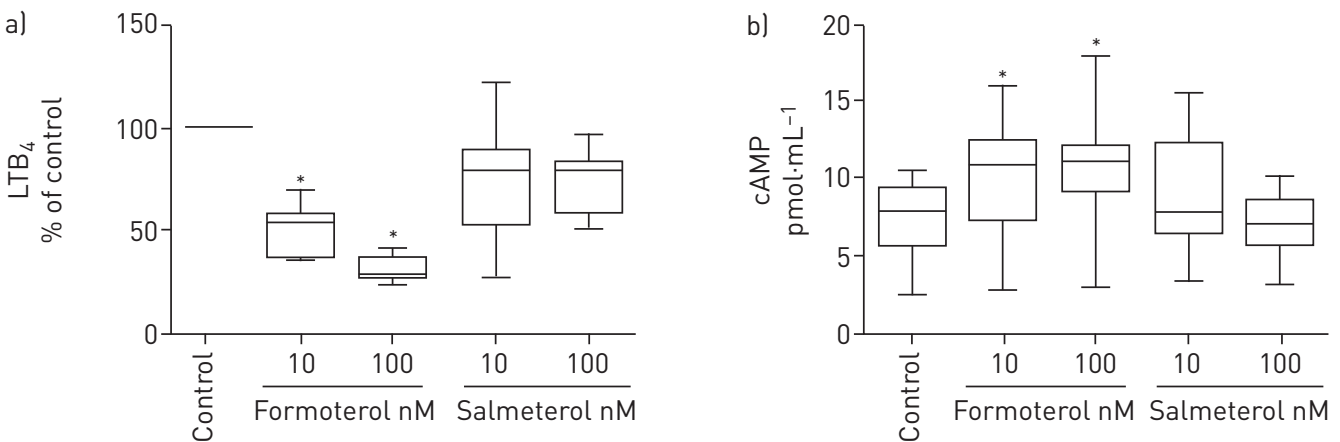

FIGURE 2 Effects of formoterol and salmeterol on the a) production of leukotriene $\mathrm{B}_{4}\left(\mathrm{LTB}_{4}\right)$ (as a percentage of that of the control) following activation of neutrophils with $\mathrm{N}$-formyl-L-methionyl-L-leucyl-L-phenylalanine (fMLP) $(1 \mu \mathrm{M})$ and on $b$ ) intracellular cAMP ( $=5-8)$. The median $\mathrm{LTB}_{4}$ values (interquartile range) for the responses of control, drug-free, unstimulated and fMLP-activated neutrophils were $36(27-110)$ and $200(190-1667) \mathrm{pg} \cdot \mathrm{mL}^{-1}$ respectively. In the case of cAMP, the value for the drug-free control system was $7.76(6.2-9) \mathrm{pmol} \cdot \mathrm{mL}^{-1}$. *: $p<0.05$ for comparison of formoterol or salmeterol with the corresponding control systems. 
of neutrophils are differentially affected by formoterol and may also vary according to the type of inflammatory stimulus. This contention is supported by the finding, albeit in the case of salmeterol, that exposure of neutrophils to aqueous cigarette smoke in vitro in the presence of the LABA results in significant attenuation of production of CXCL8, while other inflammatory activities, such as production of ROS and degranulation, are unaffected [14]. More recently, and consistent with the findings of the current study, Wex et al. [15] reported that intranasal administration of aerosolised olodaterol, an ultra-LABA with nearly full agonist activity, to mice and guinea pigs significantly attenuated pulmonary neutrophilia induced by inhalation of cigarette smoke or LPS. These anti-inflammatory actions of olodaterol were sustained following repeated dosing over 4 days and were associated with significant decreases in the concentrations of an array of proinflammatory cytokines/chemokines in bronchoalveolar lavage fluid, as well as inhibition of expression of the $\beta_{2}$-integrin CD11b.

With respect to possible clinical relevance of the findings of the current study, only a few large randomised trials have compared the efficacy of the $\mathrm{F} / \mathrm{B}$ and $\mathrm{S} / \mathrm{F}$ combinations in patients with COPD. The recent PATHOS trial retrospectively compared the efficacy of $S / F$ with that of $F / B$ on the rates of exacerbation during treatment of COPD patients in Sweden [16]. The results of this trial indicated that F/B inhalation therapy was more effective than S/F, while a smaller trial from Canada reported similar results to those of the PATHOS study [17]. Notwithstanding differences between these two formulations, such as efficacy of delivery to the lower airways and lipophilicity of fluticasone, the apparently superior anti-inflammatory efficacy of $\mathrm{F} / \mathrm{B}$ in COPD, which has also been described in asthma [18-20], may be related to the potency of the interaction of formoterol with $\beta_{2}$ ARs on neutrophils relative to that of salmeterol.

Clearly, studies of the type described here, which have focused on circulating neutrophils from healthy individuals, could serve as a template for future studies encompassing patients with COPD. Such studies would, however, require careful attention to: 1) inclusion of airway as well as circulating neutrophils; 2) awareness of the possible existence of a neutrophil phenotype that exhibits a high level of pro-inflammatory activity under the hypoxic conditions associated with COPD [1]; 3) consideration that COPD is not a homogeneous disease entity but rather a collection of phenotypes with potentially differential effects on neutrophil reactivity; and 4) interpretational difficulties posed by the complicating effects of existing anti-inflammatory therapies.

In conclusion, formoterol at therapeutically relevant concentrations, but not salmeterol, effectively suppresses neutrophil reactivity in vitro, an activity that, if operative in the clinical setting of COPD, may contribute to anti-inflammatory efficacy when used in combination with ICS.

Gregory R. Tintinger ${ }^{1,2}$, Annette J. Theron ${ }^{2,3}$, Helen C. Steel ${ }^{2}$, Charles Feldman ${ }^{4}$ and Ronald Anderson ${ }^{2}$ ${ }^{1}$ Department of Internal Medicine, Faculty of Health Sciences, University of Pretoria, Pretoria, South Africa. ${ }^{2}$ Institute for Cellular and Molecular Medicine, Department of Immunology, Faculty of Health Sciences, University of Pretoria, Pretoria, South Africa. ${ }^{3}$ Tshwane Academic Division of the National Health Laboratory Service, Pretoria, South Africa. ${ }^{4}$ Division of Pulmonology, Department of Internal Medicine, Faculty of Health Sciences, University of the Witwatersrand and Charlotte Maxeke Johannesburg Academic Hospital, Johannesburg, South Africa.

Correspondence: Gregory R. Tintinger, Dept of Internal Medicine, PO Box 667, Pretoria 0001, South Africa. E-mail: grtintinger@gmail.com

Received: April 152015 | Accepted after revision: July 262015

Support statement: G.R. Tintinger and C. Feldman are supported by the National Research Foundation of South Africa, and R. Anderson by the South African Medical Research Council. Funding information for this article has been deposited with FundRef.

Conflicts of interest: Disclosures can be found alongside this article at openres.ersjournals.com

@ERSpublications

Formoterol suppresses neutrophil reactivity in vitro; in COPD, this may contribute to antiinflammatory efficacy http://ow.ly/Qr9fE

The content of this work is ( ) the authors or their employers. Design and branding are @ERS 2015. This article is open access and distributed under the terms of the Creative Commons Attribution Non-Commercial Licence 4.0. 


\section{References}

1 Hoenderdos K, Condliffe A. The neutrophil in chronic obstructive pulmonary disease. Am J Respir Cell Mol Biol 2013; 48: 531-539.

2 Kew KM, Dias S, Cates CJ. Long-acting inhaled therapy (beta-agonists, anticholinergics and steroids) for COPD: a network meta-analysis. Cochrane Database Syst Rev 2014; 3: CD010844.

3 Calverley PMA, Anderson JA, Celli B, et al. Salmeterol and fluticasone propionate and survival in chronic obstructive pulmonary disease. N Engl J Med 2007; 8: 775-789.

4 Sharafkhaneh A, Mattewal AS, Abraham VM, et al. Budesonide/formoterol combination in COPD: a US perspective. Int J Chron Obstruct Pulmon Dis 2010; 5: 357-366.

5 Barnes PJ. New molecular targets for the treatment of neutrophilic diseases. J Allergy Clin Immunol 2007; 119: $1055-1062$.

6 Gravett CM, Theron AJ, Steel HC, et al. Interactive inhibitory effects of formoterol and montelukast on activated human neutrophils. Eur Respir J 2010; 36: 1417-1424.

7 Anderson R, Theron AJ, Steel HC, et al. The beta-2-adrenoreceptor agonists, formoterol and indacaterol, but not salbutamol, effectively suppress the reactivity of human neutrophils in vitro. Mediators Inflamm 2014; 2014: 105420.

8 de Coupade C, Gear RW, Dazin PF, et al. $\beta_{2}$-Adrenergic receptor regulation of human neutrophil function is sexually dimorphic. Br J Pharmacol 2004; 143: 1033-1041.

9 Theron AJ, Steel HC, Tintinger GR, et al. Can the anti-inflammatory activities of $\beta 2$-agonists be harnessed in the clinical setting? Drug Des Devel Ther 2013; 7: 1387-1398.

10 Profita M, Bonanno A, Montalbano AM, et al. $\beta_{2}$ long-acting and anticholinergic drugs control TGF- $\beta 1$-mediated neutrophilic inflammation in COPD. Biochim Biophys Acta 2012; 1822: 1079-1089.

11 Kato G, Takahashi $\mathrm{K}$, Tashiro $\mathrm{H}$, et al. $\beta 2$ adrenergic agonist attenuates house dust mite-induced allergic airway inflammation through dendritic cells. BMC Immunol 2014; 15: 39.

12 Lambers C, Qi Y, Eleni P, et al. Extracellular matrix composition is modified by $\beta_{2}$-agonists through cAMP in COPD. Biochem Pharmacol 2014; 91: 400-408.

13 Strandberg K, Blidberg K, Sahlander K, et al. Effect of formoterol and budesonide on chemokine release, chemokine receptor expression and chemotaxis in human neutrophils. Pulm Pharmacol Ther 2010; 23: 316-323.

14 Mortaz E, Rad MV, Johnson M, et al. Salmeterol with fluticasone enhances the suppression of IL-8 release and increases the translocation of glucocorticoid receptor by human neutrophils stimulated with cigarette smoke. $J \mathrm{Mol}$ Med 2008; 86: 1045-1056.

15 Wex E, Kollak I, Duechs MJ, et al. The long-acting $\beta_{2}$-agonist olodaterol attenuates pulmonary inflammation. Br J Pharmacol 2015; 172: 3537-3547.

16 Larsson K, Janson C, Lisspers K, et al. Combination of budesonide/formoterol more effective than fluticasone/ salmeterol in preventing exacerbations in chronic obstructive pulmonary disease: the PATHOS study. J Intern Med 2013; 273: 584-594.

17 Blais L, Forget A, Ramachandran S. Relative effectiveness of budesonide/formoterol and fluticasone propionate/ salmeterol in a 1-year, population-based, matched cohort study of patients with chronic obstructive pulmonary disease (COPD): effect on COPD-related exacerbations, emergency department visits and hospitalizations, medication utilization, and treatment adherence. Clin Ther 2010; 32: 1320-1328.

18 Akamatsu T, Shirai T, Kato M, et al. Switching from salmeterol/fluticasone to formoterol/budesonide combinations improves peripheral airway/alveolar inflammation in asthma. Pulm Pharmacol Ther 2014; 27 : 52-56.

19 Hozawa S, Terada M, Hozawa M. Comparison of the effects of budesonide/formoterol maintenance and reliever therapy with fluticasone/salmeterol fixed-dose treatment on airway inflammation and small airway impairment in patients who need to step-up from inhaled corticosteroid monotherapy. Pulm Pharmacol Ther 2014; 27: 190-196.

20 Hojo M, Iikura M, Hirashima J, et al. A comparison of long-term anti-inflammatory effect of two ICS/LABA combination inhalers; fix-dosed maintenance therapy with budesonide/formoterol and salmeterol/fluticasone. Allergol Int 2014; 63: 103-111. 